Mathematical Research Letters 1, 49-52 (1994)

\title{
GENERAL HYPERPLANE SECTIONS OF NONSINGULAR FLOPS IN DIMENSION 3
}

\author{
YUJIRO KAWAMATA
}

Let $X$ be a 3 -dimensional complex manifold, and $f: X \rightarrow Y$ a proper bimeromorphic morphism to a normal complex space which contracts an irreducible curve $C \subset X$ to a singular point $Q \in Y$ while inducing an isomorphism $X \backslash C \simeq Y \backslash\{Q\}$. We assume that the intersection number with the canonical divisor $\left(K_{X} \cdot C\right)$ is zero. In this case, it is known that the singularity of $Y$ is Gorenstein terminal, and there exists a flop $f^{\#}$ : $X^{\#} \rightarrow Y([\mathrm{R}])$, which we call a nonsingular flop because $X$ is nonsingular.

In order to investigate $f$ analytically, we replace $Y$ by its germ at $Q$ and consider a general hyperplane section $H$ of $Y$ through $Q$. Then $H$ has only a rational double point, its pull-back $L \subset X$ by $f$ is normal, and the induced morphism $f_{H}: L \rightarrow H$ factors the minimal resolution $g: M \rightarrow H$ ([R]). The dual graph $\Gamma$ of the exceptional curves of $g$ is a Dynkin diagram of type $A_{n}, D_{n}$ or $E_{n}$. Let $F=\sum_{k=1}^{n} m_{k} C_{k}$ be the fundamental cycle for $g$ on $M$. The natural morphism $h: M \rightarrow L$ is obtained by contracting all the exceptional curves of $g$ except the strict transform $C_{k_{0}}$ of $C$.

Kollár defined an invariant of $f$ called the length as the length of the scheme theoretic fiber $f^{-1}(Q)$ at the generic point of $C$. It coincides with the multiplicity $m_{k_{0}}$ of the fundamental cycle at $C_{k_{0}}$.

Katz and Morrison proved the following theorem ([KM, Main Theorem]). The purpose of this paper is to give its simple geometric proof.

Theorem. Let $f: X \rightarrow Y$ be as above. Then the singularity of the general hyperplane section $H$ and the partial resolution $f_{H}: L \rightarrow H$ are determined by the length $\ell$ of $f$. More precisely, $H$ has a rational double point of type $A_{1}, D_{4}, E_{6}, E_{7}, E_{8}$, or $E_{8}$, if $\ell=1,2,3,4,5$ or 6 , respectively.

We note that there is only one irreducible component of $g^{-1}(Q)$ whose multiplicity in $F$ coincides with $\ell$ in the above cases.

Proof. Let $H^{\prime}$ be another general hyperplane section of $Y$ through $Q$, and $f_{H^{\prime}}: L^{\prime} \rightarrow H^{\prime}$ the induced morphism. $H$ and $H^{\prime}$ have the same type of

Received October 6, 1993. 
singularities and so do $L$ and $L^{\prime}$. Let $P_{i}$ and $P_{i}^{\prime}$ be the singular points of $L$ of $L^{\prime}$, respectively.

Let $D$ be the effective Cartier divisor on $L$ given by $L^{\prime} \cap L$. Then $D$ is a general member of the linear system of effective Cartier divisors on $L$ which contain $C$ and such that $(D \cdot C)=0$. In fact, if $s_{0}$ is the global section of $\mathcal{O}_{L}(-C) \subset \mathcal{O}_{L}$ corresponding to $D$, then from an exact sequence

$$
0 \rightarrow \mathcal{O}_{X}(-L) \rightarrow \mathcal{O}_{X} \rightarrow \mathcal{O}_{L} \rightarrow 0
$$

there exists a lifting $s \in H^{0}\left(X, \mathcal{O}_{X}\right)$ of $s_{0}$ which defines $L^{\prime}$, because $H^{1}\left(X, \mathcal{O}_{X}(-L)\right)=0$.

Let $\tilde{D}$ be the total transform of $D$ on $M$. Then we can write $\tilde{D}=F+D^{\prime}$ for some $D^{\prime}$ which is reduced, nonsingular and has no common irreducible components with $F$. If $\Gamma$ is of type $A_{n}$, then $D^{\prime}$ has 2 irreducible components each of which intersects transversally one of the end components of $F$. Otherwise, $D^{\prime}$ is irreducible, and intersects transversally a uniquely determined component $C_{k_{1}}$ of $F$, for which $m_{k_{1}}=2$ holds $\left(k_{1}\right.$ may be equal to $\left.k_{0}\right)$.

Let $t$ be the global section of $\mathcal{O}_{X}$ corresponding to $L$. Then $s+c t$ is also a lifting of $s_{0}$ for any $c \in \mathbb{C}$. Let $L^{\prime}(c)$ be the corresponding divisor on $X$.

Let $P$ be a point on $C$ which is different from the $P_{i}$. For local analytic coordinates $\{x, y, t\}$, we can write $s+c t=F(x, y)+t(G(x, y, t)+c)$. For a general choice of $c,\left.(G(x, y, t)+c)\right|_{C}$ does not vanish at the singular points $P_{i}^{\prime}$ of $L^{\prime}=L^{\prime}(0)$ other than the $P_{i}$, and has only simple zeroes at some points $P_{j}^{\prime \prime}$. Then $L^{\prime \prime}=L^{\prime}(c)$ has singularities only at the $P_{j}^{\prime \prime}$, besides possibly at the $P_{i}$, with equations of the type $x^{\ell}+t y=0$.

If we replace $L^{\prime}$ by $L^{\prime \prime}$, we conclude that $L^{\prime}$ has only singularities of type $A_{\ell-1}$ outside the $P_{i}$. We shall investigate the singularities of $L^{\prime}$ at the $P_{i}$ case by case.

Let $\Gamma_{i}$ be the dual graph of the exceptional curves of $h$ over $P_{i}$, and $F_{i}$ the corresponding fundamental cycle. From the description of $\tilde{D}$ above, we can calculate the multiplicity $d_{i}$ of $D$ at the point $P_{i}$ by $d_{i}=\left(\left(m_{k_{0}} C_{k_{0}}+D^{\prime}\right) \cdot F_{i}\right)$.

If $\ell=1$ or 2 , then we can check that $d_{i} \leq 3$. If 2 singular surfaces meet at a common singular point, then the intersection curve has multiplicity at least 4 there. Hence $L^{\prime}$ is nonsingular at the $P_{i}$. Then it follows that $\Gamma=A_{1}$ or $D_{4}$, respectively.

But if $\ell \geq 3$, then $d_{i}$ can be bigger, and we should look at the singularity of $L^{\prime}$ more closely.

We assume first that $\ell=3$. If $\Gamma=E_{6}$, then there is nothing to prove. We have to prove that $\Gamma \neq E_{7}, E_{8}$. If $\Gamma=E_{7}$, then $L$ has two singular points $P_{1}$ and $P_{2}$, where $F_{1}$ meets $D^{\prime}$. We have 2 cases; $\Gamma_{1}=A_{1}$ and 
$\Gamma_{2}=A_{5}$, or $\Gamma_{1}=A_{4}$ and $\Gamma_{2}=A_{2}$. In the former case, $L^{\prime}$ has at most $A_{1}$ singularity at $P_{1}$ because of the symmetry of $L$ and $L^{\prime}$, while being nonsingular at $P_{2}$, since $d_{2}=3$. Therefore, $L^{\prime}$ has simpler singularities than $L$, a contradiction. In the latter case, it has at most $A_{2}$ at $P_{2}$. We shall prove that $L^{\prime}$ has $A_{1}$ at $P_{1}$.

Let $\mu: X^{(1)} \rightarrow X$ be the blowing-up at $P_{1}, E \simeq \mathbb{P}^{2}$ the exceptional divisor, and $L^{(1)}$ (resp. $\left.L^{(1) \prime}\right)$ the strict transform of $L$ (resp. $\left.L^{\prime}\right) . \quad B=$ $L^{(1)} \cap E$ consists of 2 lines $B_{1}$ and $B_{2}$ which correspond to the 2 end components of $F_{1}$. Their multiplicities in $\mu^{*} L^{\prime} \cap L^{(1)}$ are equal to those in $F$, which are 2. If $L^{\prime}$ has multiplicity $n$ at $P_{1}$, then we have $\mu^{*} L^{\prime}=L^{(1) \prime}+n E$ and $L^{(1) \prime} \cap E$ is a plane curve of degree $n$. Since $L^{(1)} \cdot E=B$, we deduce that $\mu^{*} L^{\prime}=L^{(1) \prime}+2 E$, and neither of the $B_{i}$ are contained in $B^{\prime}=L^{(1) \prime} \cap E$. Thus the intersection of 2 conics $B$ and $B^{\prime}$ is equal to $\left(L^{(1)} \cap L^{(1) \prime}\right) \cap E$. We see from the description of $\tilde{D}$ that it consists of 2 points, one at $B_{1} \cap B_{2}$ and the other on one component $B_{1}$. Then $B^{\prime}$ must be a nonsingular conic, and $L^{\prime}$ has $A_{1}$ singularity at $P_{1}$.

If $\Gamma=E_{8}$, then we have again 2 cases; $\Gamma_{1}=A_{1}$ and $\Gamma_{2}=E_{6}$, or $\Gamma_{1}=A_{7}$. In the former case, $L^{\prime}$ has at most $A_{1}$ singularity at $P_{1}$, while being nonsingular at $P_{2}$, since $d_{2}=3$. In the latter case, it has $A_{1}$ at $P_{1}$ as in the case of $E_{7}$.

Next we assume that $\ell=4$. If $\Gamma=E_{7}$, then there is nothing to prove. If $\Gamma=E_{8}$, then we have 2 cases: $\Gamma_{1}=D_{5}$ and $\Gamma_{2}=A_{2}$, or $\Gamma_{1}=A_{6}$ and $\Gamma_{2}=A_{1}$.

In the former case, $L^{\prime}$ has at most $A_{2}$ singularity at $P_{2}$. By the symmetry of $L$ and $L^{\prime}, L^{\prime}$ has $D_{5}$ at $P_{1}$. Let $\mu: X^{(1)} \rightarrow X, E, L^{(1)}$ and $L^{(1) \prime}$ as before. $B=L^{(1)} \cap E$ is a line, and $L^{(1)} \cdot E=2 B$. Since the corresponding curve has multiplicity 4 in $F$, we have $\mu^{*} L^{\prime}=L^{(1) \prime}+2 E$, and $B$ is not contained in $B^{\prime}=L^{(1) \prime} \cap E$. $L^{(1)}$ has 2 singular points $P_{1}^{(1)}$ and $P_{2}^{(1)}$ on $B$ which are of types $A_{3}$ and $A_{1}$, respectively. We have $B \cap B^{\prime}=P_{1}^{(1)}$ by the description of $\tilde{D}$.

Let $\nu: X^{(2)} \rightarrow X^{(1)}$ be the blowing-up at $P_{1}^{(1)}, E^{(1)} \simeq \mathbb{P}^{2}$ the exceptional divisor, and $L^{(2)}$ (resp. $L^{(2) \prime}$ ) the strict transform of $L^{(1)}$ (resp. $L^{(1) \prime}$ ). $B^{(1)}=L^{(2)} \cap E^{(1)}$ consists of 2 lines, and one of the corresponding curves on $M$ has multiplicity 3 in $F$, hence $\nu^{*} L^{(1) \prime}=L^{(2) \prime}+E^{(1)}$, and $L^{(1) \prime}$ is nonsingular at $P_{1}^{(1)}$. But this contradicts the symmetry of $L$ and $L^{\prime}$.

In the latter case, $L^{\prime}$ has at most $A_{1}$ singularity at $P_{2}$. Let $\mu: X^{(1)} \rightarrow X$, etc., as before. $B=L^{(1)} \cap E$ consists of 2 lines $B_{1}$ and $B_{2}$, which correspond to the 2 end components of $F_{1}$. Since their multiplicities in $F$ are 3 and 2, $B_{1}$ is contained in $B^{\prime}=L^{(1) \prime} \cap E$, while $B_{2}$ is not. Thus we have $B^{\prime}=B_{1}+B_{2}^{\prime}$ with $B_{2} \neq B_{2}^{\prime}$. Since $C$ is nonsingular, its strict transform 
on $X^{(1)}$ intersects $E$ at only one point. It is also the image of $C_{k_{0}}$ on $X^{(1)}$, so it passes through the point $B_{1} \cap B_{2}$, hence not $B_{1} \cap B_{2}^{\prime}$, a contradiction to the symmetry.

Finally, if $\ell \geq 5$, the assertion of the theorem is clear. Q.E.D.

\section{References}

[KM] S. Katz and D. R. Morrison, Gorenstein threefold singularities with small resolutions via invariant theory of Weyl groups, J. Alg. Geom. 1, 449-530.

[R] M. Reid, Minimal models of canonical 3-folds, Adv. St. Pure Math. 1 (1983), $131-180$.

Department of Mathematichl Sciences, University of Tokyo, Hongo, B UNKYO, TOKYO, 113 , JAPAN

E-mail address: kawamata@math.s.u-tokyo.ac.jp 